ISSN 2073-4336

www.mdpi.com/journal/games

Article

\title{
An Experimental Analysis of Asymmetric Power in Conflict Bargaining
}

\author{
Katri Sieberg ${ }^{1}$, David Clark ${ }^{2}$, Charles A. Holt ${ }^{3}$, Timothy Nordstrom ${ }^{4}$ and William Reed ${ }^{5, *}$ \\ 1 School of Social Science and Humanities, FIN-33014 University of Tampere, Finland; \\ E-Mail: katri.sieberg@uta.fi \\ 2 Department of Political Science, Binghamton University (SUNY), Binghamton, NY 13902, USA; \\ E-Mail: dclark@binghamton.edu \\ 3 Department of Economics, University of Virginia. Charlottesville, VA 22904, USA; \\ E-Mail: holt@virginia.edu \\ 4 Department of Political Science, The University of Mississippi, Oxford, MS 38677, USA; \\ E-Mail: tnordstr@olemiss.edu \\ 5 Department of Government \& Politics, University of Maryland, 3140 Tydings Hall, College Park, \\ MD 20742, USA
}

* Author to whom correspondence should be addressed; E-Mail: wlr@ umd.edu; Tel. 301-405-4156.

Received: 12 June 2013; in revised form: 9 July 2013 / Accepted: 24 July 2013 /

Published: 2 August 2013

\begin{abstract}
Demands and concessions in a multi-stage bargaining process are shaped by the probabilities that each side will prevail in an impasse. Standard game-theoretic predictions are quite sharp: demands are pushed to the precipice with nothing left on the table, but there is no conflict regardless of the degree of power asymmetry. Indeed, there is no delay in reaching an agreement that incorporates the (unrealized) costs of delay and conflict. A laboratory experiment has been used to investigate the effects of power asymmetries on conflict rates in a two-stage bargaining game that is (if necessary) followed by conflict with a random outcome. Observed demands at each stage are significantly correlated with power, as measured by the probability of winning in the event of disagreement. Demand patterns, however, are flatter than theoretical predictions, and conflict occurs in a significant proportion of the interactions, regardless of the degree of the power asymmetry. To address these deviations from the standard game-theoretic predictions, we also estimated a logit quantal response model, which generated the qualitative patterns that are observed in the data. This one-parameter generalization of the Nash equilibrium permits a
\end{abstract}


deconstruction of the strategic incentives that cause demands to be less responsive to power asymmetries than Nash predictions.

Keywords: bargaining; conflict; quantal response equilibrium; laboratory experiments

\section{Introduction}

When John von Neumann and Oscar Morgenstern began working on Theory of Games and Economic Behavior almost a century ago, they were motivated by the observation that most received theory pertained to price-based interactions in large markets, whereas a large fraction of economic activity involves bargaining in small group settings. The only economics class that John Nash took at Carnegie-Mellon as an undergraduate was International Trade, and he was intrigued by the absence of a widely accepted model for negotiations between countries over trade issues. The seminal work of these game theorists and others has generated a large bargaining literature, involving both theory and applications to specific social sciences. ${ }^{1}$ Over the past two decades, for example, students of world politics have increasingly thought of interstate war as a bargaining problem. Drawing heavily on the literature of economics, bargaining models have become commonplace and have provided a number of significant insights regarding when and why states resort to arms. An analysis of strategic bargaining has been used to explain how wars begin and end, and how long they last. In addition, bargaining models have been adapted to explain the durability of peace agreements, extended deterrence, and the relation between trade and conflict. This paradigm also extends to other issues of importance to social scientists, e.g., the incidence of costly strikes that follow breakdowns in labor negotiations, or costly delays in the adjudication of tort claims. In all of these examples, bargaining is conducted in the "shadow of power" [14]. In particular, a failure to reach an agreement in these settings produces a crisis or trial that is settled by a contest with an uncertain outcome.

An alternative perspective on conflict is that stable situations are sometimes disrupted by "exogenous" demands that arise from overconfidence, perceived or actual inequities, and the personalities of leaders that come to power. Bargaining models, in contrast, are focused on structural factors, e.g., power asymmetries, that determine strategic demands and the conflicts that arise endogenously in this framework [5,14,15,19]. These scholars claim that parties evaluate the distribution of benefits in light of the distribution of power, and decide whether to make demands about reallocating benefits to better represent the realities of power, as represented by the probabilities of winning in a conflict. So states of equal power, who also have equal shares in the benefits of the international system, are less likely to fight than are equally powerful states with decidedly unequal benefits. Similarly, the relative probabilities of winning in a labor dispute should affect strike decisions

\footnotetext{
${ }^{1}$ See Holt and Roth [10] for specific references and a perspective on motivations for early work on game theory and on the Nash equilibrium in particular. The finite-stage bargaining models considered in this paper can be solved by backward induction, using Reinhard Selten's notion of subgame perfection to rule out Nash equilibria that involve non-credible threats to reject favorable offers.

${ }^{2}$ Numerous variants of the bargaining model arise in the political science literature. We focus on a two-stage version of the model analyzed in Fearon [5].
} 
made by employees. Both the amount of the challenger's demand and the probability of bargaining failure arise from the imbalance between the probability of winning in a conflict and the distribution of benefits ex ante (Reed, Clark, Nordstrom, Hwang 2008).

Despite the theoretical appeal of the basic insights from bargaining theory, empirical evaluation of the claims that arise from these models has lagged behind. The lack of empirical work is not for lack of effort. Instead, the gap is due to the simple fact that many elements of the basic bargaining model are either difficult to measure or are unobservable. For example, in international bargaining and in labor negotiations alike, neither the probability of success nor the costs of bargaining failure are as explicitly known as models would suggest. Experimental analysis offers a unique opportunity to observe how power imbalances and other factors affect behavior in the context of models with clear theoretical predictions.

A common modeling approach is to constrain the timing and nature of decisions in a manner that makes it possible to derive clear theoretical predictions. The structure of a bargaining process can be simplified by designating one person as a decision maker in each discrete stage of the game, e.g., with alternating offers or a randomly determined decision order. The standard Stahl-Rubinstein model of alternating-offer bargaining has an infinite horizon, with no conflict, but effects of discounting and delay are sufficient to induce an immediate agreement that is shaped by the potential costs of delay. Finite versions of this model that are convenient for laboratory experiments typically impose exogenous payoffs in the final stage. ${ }^{3}$ For example, the single-stage ultimatum game that has been used in hundreds of laboratory experiments has zero payoffs for each player if the ultimatum demand by one is rejected by the other. A zero payoff is sensible if a monopolist walks away from a deal, leaving both parties with no trade, or if conflict involves a total destruction of the potential surplus. Conflict, whether it is legal, economic, or military, can involve significant costs, but in the absence of nuclear escalation, these costs are unlikely to eradicate the value of the rights being contested. Moreover, conflict involves additional uncertainty about who will ultimately prevail.

In a laboratory experiment, conflict risk can be implemented by having the outcome of an impasse be determined by a random lottery. The predictions of this conflict bargaining model with a lottery depend on relative power, as measured by the probabilities that each side would prevail. In theory, demands and counter-offers should be shaped by power asymmetries, along with delay and conflict costs, but the subgame-perfect Nash prediction is similar to that of other models: (1) no delay; (2) nothing left on the table (in an expected payoff sense); and (3) no conflict. Un-modeled "exogenous" factors like overconfidence, overweighting of low probabilities, or differences in risk aversion and perceptions of fairness can generate conflict in laboratory bargaining games.

Our goal in this paper is to evaluate the extent to which bargaining outcomes and conflict rates are shaped by structural factors like power asymmetries. Even though the theory is straightforward, there is good reason to be skeptical of predictions that rely on backward induction reasoning. Our analysis therefore, is based on a laboratory experiment with financially motivated human subjects. In short, we find a significant relationship between players' known winning probabilities and their initial demands and counteroffers. The effects of power asymmetries, however, are not as strong as predicted, and

\footnotetext{
${ }^{3}$ Goeree and Holt [8] report experiments with an array of two-stage bargaining games with a shrinking "pie" and zero payoffs in the event of an impasse, but with asymmetric "outside earnings" that are varied to enhance fairness concerns.
} 
conflict occurs in about a quarter of the interactions, irrespective of whether or not differences in the probabilities of winning are high or low. Some behavioral explanations of the observed deviations from game-theoretic predictions are analyzed.

\section{A Simple Model of Conflict Bargaining}

We begin with a standard analysis of a two-stage model, which is followed by a derivation of the predictions for the particular setup parameters used in the experiment. In the model, two risk neutral players bargain over the division of surplus of size $S$, e.g., a parcel of territory or the surplus available in a trade agreement. The status quo division is $(q, S-q)$, where $q$ is the initial allocation for the first player, who begins by demanding an amount $x_{1}$ and offering $S-x_{1}$ to the other player. The responder can either accept the offer, in which case it is implemented, or make a counteroffer to divide the discounted value of the second-stage "pie," $\delta S$, where $\delta \leq 1$. Thus the counteroffer is a split of $\delta S-x_{2}$ for the first player and $x_{2}$ for the second. If the initial demand is rejected and a counteroffer is made, then the first player must decide whether to accept the counteroffer or to engage in a costly conflict, which the first player wins with probability $P$. The conflict costs for the first player ("proposer") and the second player ("responder") are denoted by $c_{p}$ and $c_{r}$ respectively.

Assuming that both the players are fully informed of the payoffs and the win probabilities, the game is solved through backward induction. In the second stage, the responder's demand would be an amount $x_{2}$ for which the residual available to the proposer, $\delta S-x_{2}$, is equal to the proposer's expected payoff in a conflict after incurring the conflict cost, $P \delta S-c_{p}$, assuming that indifference will result in acceptance. ${ }^{4}$ This equation determines the responder's second stage demand: $x_{2}=(1-P) \delta S+c_{p}$, which is a decreasing linear function of the proposer's win probability. This value of $x_{2}$ is what the responder can expect to earn if play goes to the second stage, so the proposer makes a minimal offer of this amount to the responder in the first stage:

$$
x_{1}=S-x_{2}=S-(1-P) \delta S-c_{p}=(1-\delta) S+P \delta S-c_{p}
$$

which is an increasing linear function of $P$. For example, if conflict costs are $2, S=10$, and $\delta S=9$, then the initial and final demands would be: $x_{1}=9 P-1$, and $x_{2}=11-9 P$. The effects of the payoff parameters are intuitive. For example, as delay costs increase (via a reduction in $\delta$ ) the initial demand in Equation (1) is predicted to increase to take advantage of the strategic first-mover advantage. One interesting asymmetry for this two-stage game is that the equilibrium demands depend only on the conflict cost for the proposer, and that a higher proposer conflict cost increases the predicted spread between $x_{1}$ and $x_{2}$.

\footnotetext{
4 The assumption of acceptance in the case of indifference is innocuous in a continuous model where "small" adjustments are possible. In a game with discrete decisions, this assumption could be motivated by a small aversion to risk. Alternatively, the probabilistic choice functions used for a logit model of discrete choice would imply acceptance probabilities of 0.5 in the case of indifference (see Equation (2) in Section 5 below).
} 


\section{Experimental Design}

The laboratory experiment is based on the two-stage bargaining model, with a pie size of $\$ 10$ and a second stage (discounted) pie size of $\$ 9$. The initial allocation was unbalanced at $\$ 3$ for the proposer and $\$ 7$ for the responder. Conflict costs were set at $\$ 2$ for each player, and the proposer win probabilities were set at one of four levels: 0.2, 0.4, 0.6, and 0.8, where the probabilities were explained in terms of the roll of a 10-sided die.

Subjects in the experiment were constrained to make discrete integer dollar demands, rather than continuous amounts. In this case, the backward induction arguments still yield a pattern of initial demands that is an increasing function of proposer win probabilities. The calculations may be illustrated for the case of a proposer win probability 0.8 , for which the proposer's expected payoff from conflict is $0.8(9)-2=\$ 5.20$, so a second-stage offer of $\$ 6$ would be accepted, leaving the responder with $\$ 3$. Given this knowledge, the proposer can demand $\$ 7$ in the first stage and offer $\$ 3$ to the responder. The other three sets of equilibrium demands can be derived analogously. For win probabilities of $0.2,0.4,0.6$, and 0.8 , the first stage demands are $\$ 1, \$ 3, \$ 5$, and $\$ 7$, and the second stage demands are $\$ 9, \$ 7, \$ 5$, and $\$ 3$ respectively.

The experiment was conducted using the Political Conflict option for the web-based Veconlab Bargaining Game. ${ }^{5}$ This option implements a two-stage dispute model with an array of parameter choices. The instructions are configured automatically to match the setup parameters selected. ${ }^{6}$ Each bargaining game implemented the two-stage model described in the previous section. For the parameters used, conflict is risky since the winner receives $\$ 9$ and the loser receives nothing. After conflict costs were subtracted, conflict payments are $\$ 7$ for the winner and $-\$ 2$ for the loser.

Each session consisted of 12 subjects being randomly matched for 10 rounds, with one value of $P$ used in the first five rounds, and a switch to another value of $P$ in the final five rounds. We ran 12 sessions that used all possible treatment orders. The random pairing was chosen to avoid complications arising from repeated play with the same opponent. ${ }^{7}$ The change in the win probability treatment in the second half of each session permits a within-subjects analysis of changes in relative power. Data from the second treatment, however, could be biased by "order effects," a possibility that we will consider later. Order effects can also be interesting, since the impact of changes in power asymmetries may alter conflict rates.

\footnotetext{
${ }^{5}$ The Veconlab site can be found by a Google search for "veconlab admin". From the main menu, select Bargaining, and then select Bargaining Games. The login page for subjects can be found with a google search for "veconlab login". This web-based game and can be used for teaching in any wireless classroom, as long as students are reminded to bring laptops. A direct link to this bargaining game admin setup is: http://veconlab.econ.virginia.edu/bg/bg.php

${ }^{6}$ Instructions for the $P=0.2$ treatment are provided in the Appendix.

${ }^{7}$ For instance, in repeated interactions, subjects can seek to establish a reputation and make strong demands, or firm counter-demands. Random matching does provide a chance for subjects to gain a better understanding of the procedures and payoffs, while preserving the one-shot flavor of the interaction that is more appropriate for the study of many bargaining situations that arise in unique, non-repeated legal or political settings. Fixed matchings would be more appropriate for the study of complex strategies for repetitive bargaining situations that are common in economic markets.
} 
The 144 subjects were University of Virginia students who were paid \$6 for participating, plus half of accumulated earnings. Earnings were in the \$18-\$25 range, for a one-hour session, and were paid immediately after each session.

\section{Results}

\subsection{Aggregate Data Patterns}

The aggregate proposer demands shown in Figure 1 are increasing functions of the proposer probability of winning, but the pattern is clearly flatter than the locus of Nash predictions, which are connected by a dashed line. Moreover, demands are greater than Nash predictions at all proposer win probabilities below the highest level.

Figure 1. Average Proposer Demand by Treatment.

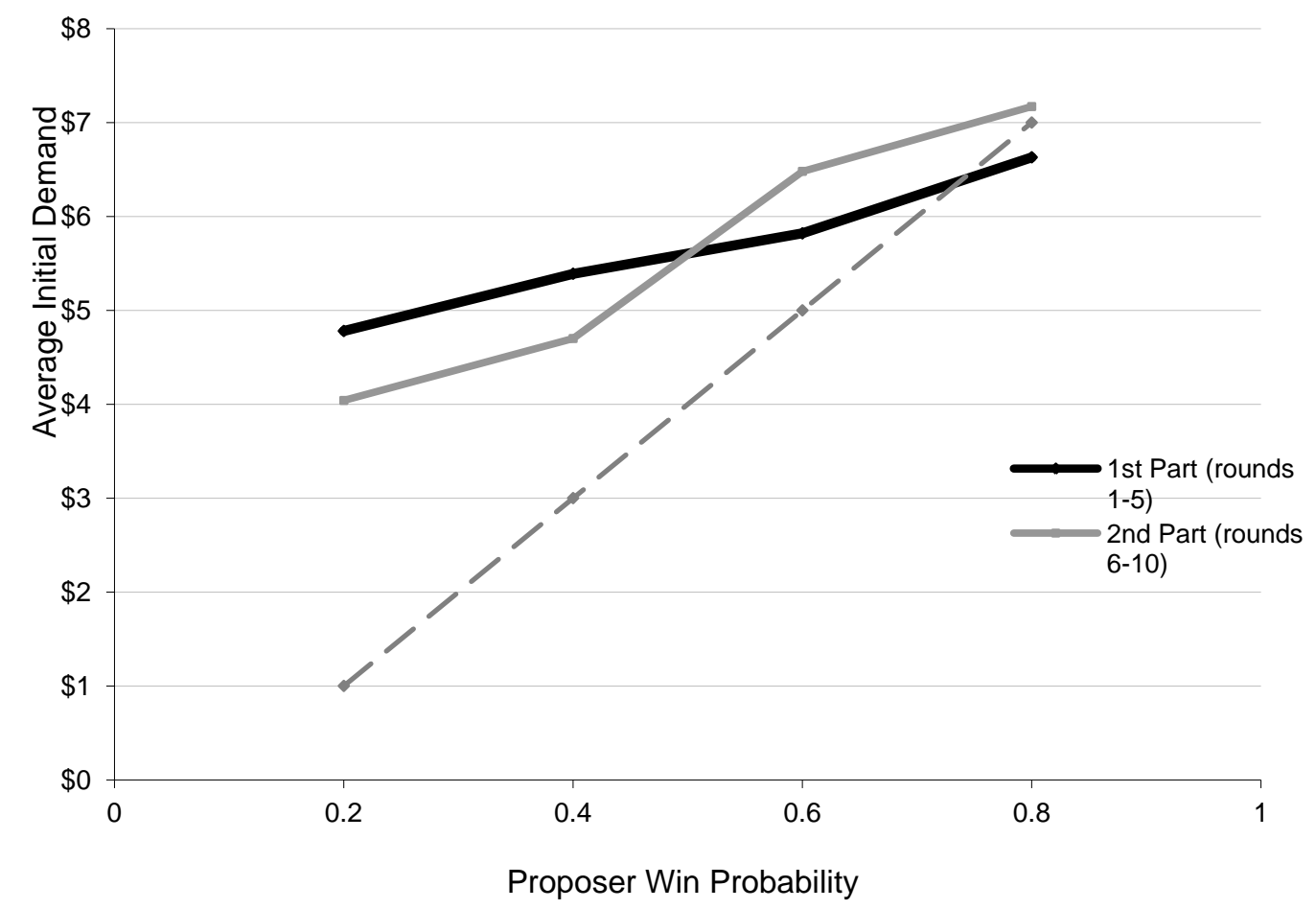

The tendency for proposers to demand more as their conflict win probabilities increase is revealed more fully in Figure 2, which shows the distributions of proposer demands for each of the four win probability treatments. These distributions shift to the right (successively thicker lines) as the value of $P$ increases.

The calculation of the Nash first-stage demand was done in a manner to ensure that it would be accepted. A demand of $\$ 1$ more than the Nash demand would always be rejected. The proposer demands in Figure 1 are, on average, in a high range where we would expect them to be rejected, at least for probabilities below 0.8. Figure 3 shows responder acceptance probabilities as a function of initial proposer demand, for each of the four proposer win probabilities, with thicker lines corresponding to treatments with higher value of $P$. Note that responder acceptance probabilities are higher than would be expected, i.e., they are often near 0.5 even when the proposer demands a dollar 
or more above the Nash prediction (of $\$ 1$ for $P=0.2, \$ 3$ for $P=0.4, \$ 5$ for $P=0.6$, and $\$ 7$ for $P=0.8$ ). The implication is that the observed tendency of proposers to make high initial demands, even when they are disadvantaged, is not irrational given the tendency for responders to accept those demands.

Figure 2. Distributions of Initial Proposer Demands by Treatment.

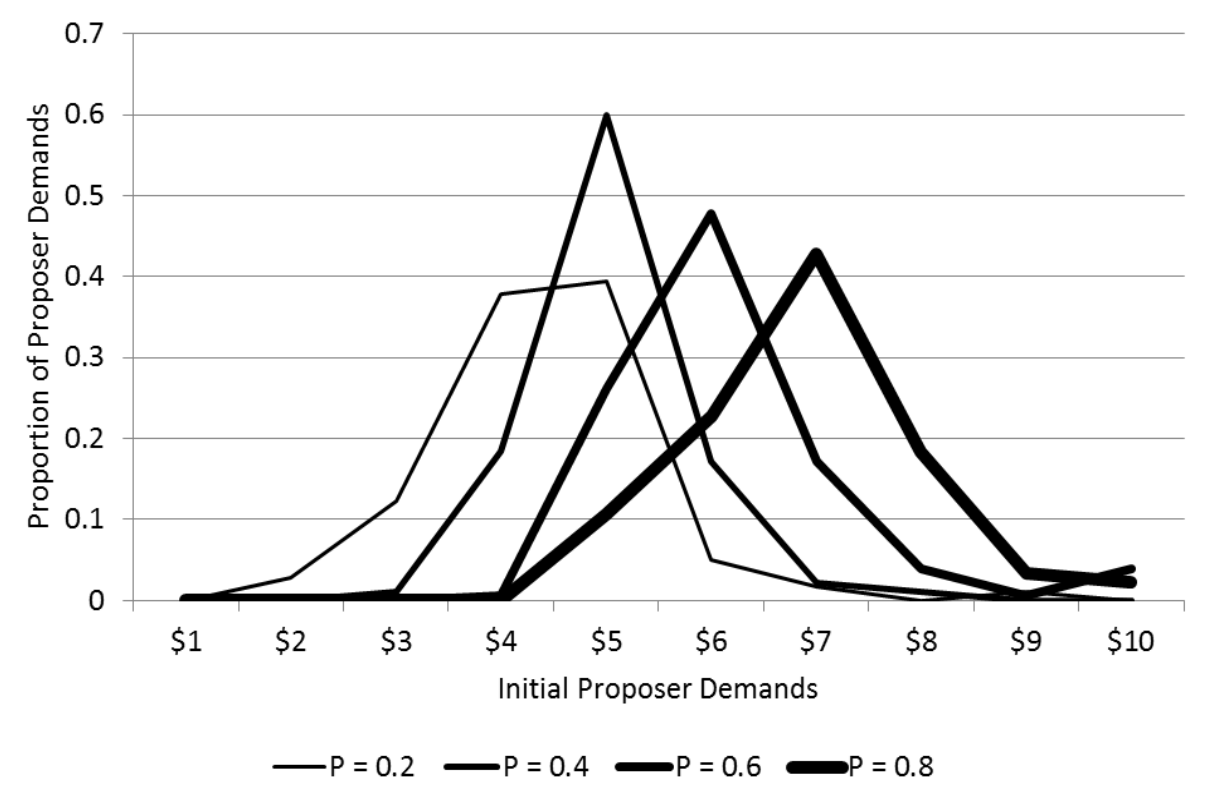

Figure 3. Responder Acceptance Probabilities as Functions of Proposer Demands.

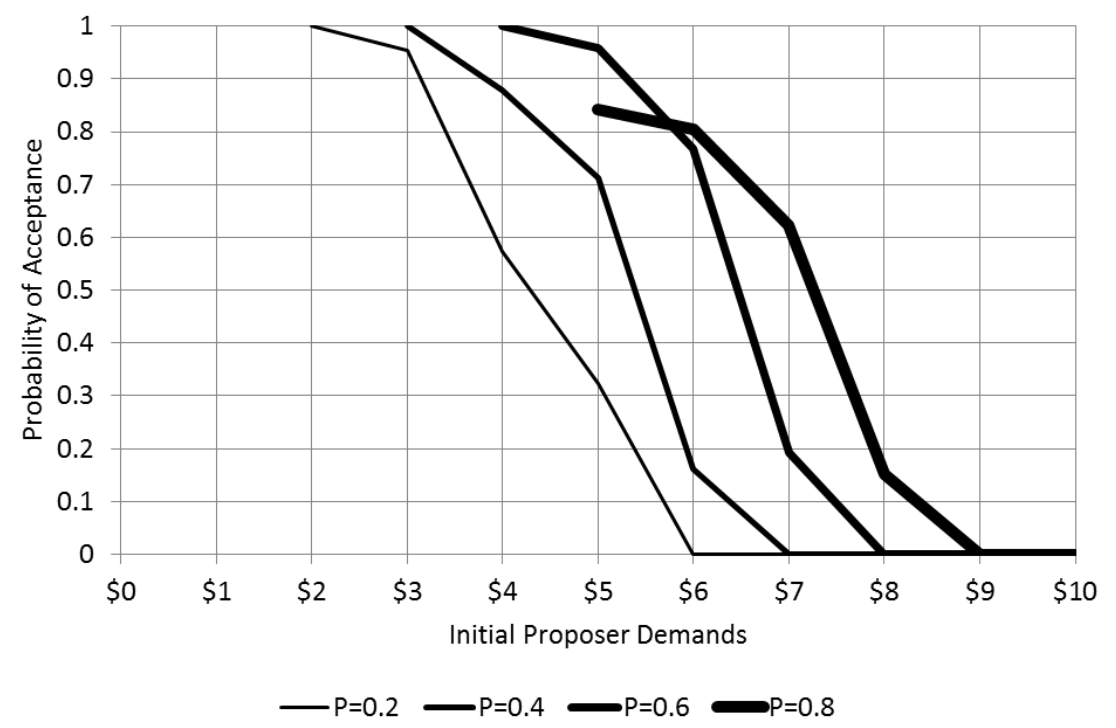

The average (second-stage) responder demands shown in Figure 4 are negatively sloped, as predicted, and are closer to Nash predictions than was the case for proposer demands. One possibility is that backward induction reasoning is more obvious in the stage closer to the possible conflict. As with proposer demands in Figure 1, there is a tendency for responder demands in Figure 4 to be flatter in the first part (rounds 1-5) and less so in the second part. The fact that responder demands are generally below the Nash predictions (except at $P=0.8$ ) suggests that some responders may have cautiously "backed off" from making the maximal demand that a risk-neutral proposer would accept. Finally, the "smoothed" nature of responder acceptance probabilities in Figure 3 indicates that these 
decisions can be modeled as being stochastic, which will be done via logit probabilistic choice functions in Section 6 below.

The proportions of conflict outcomes (for which both the proposer offer and the responder counter offer are rejected) are shown in Figure 5. Note that there is no clear relationship between conflict rates and power asymmetry, as represented by the proposer win probability. Conflict rates are lower for the second part, rounds 5-10, but these rates are much higher than Nash predictions of no conflict. Notice that conflict rates observed for the case of a high proposer win probability are slightly higher, but this difference is not statistically significant using session averages.

Figure 4. Average Responder Demand by Treatment.

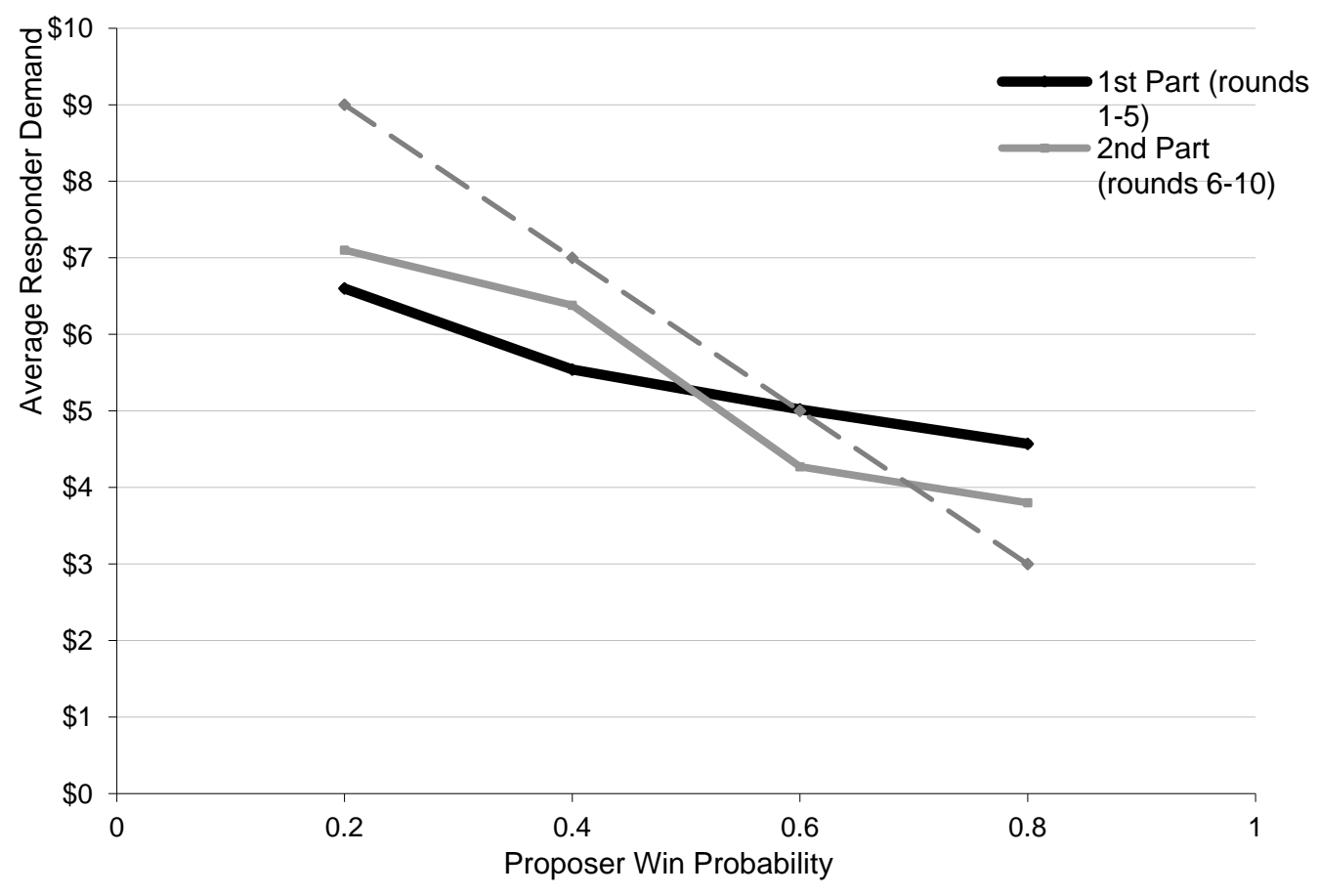

Figure 5. Conflict Rates by Treatment.

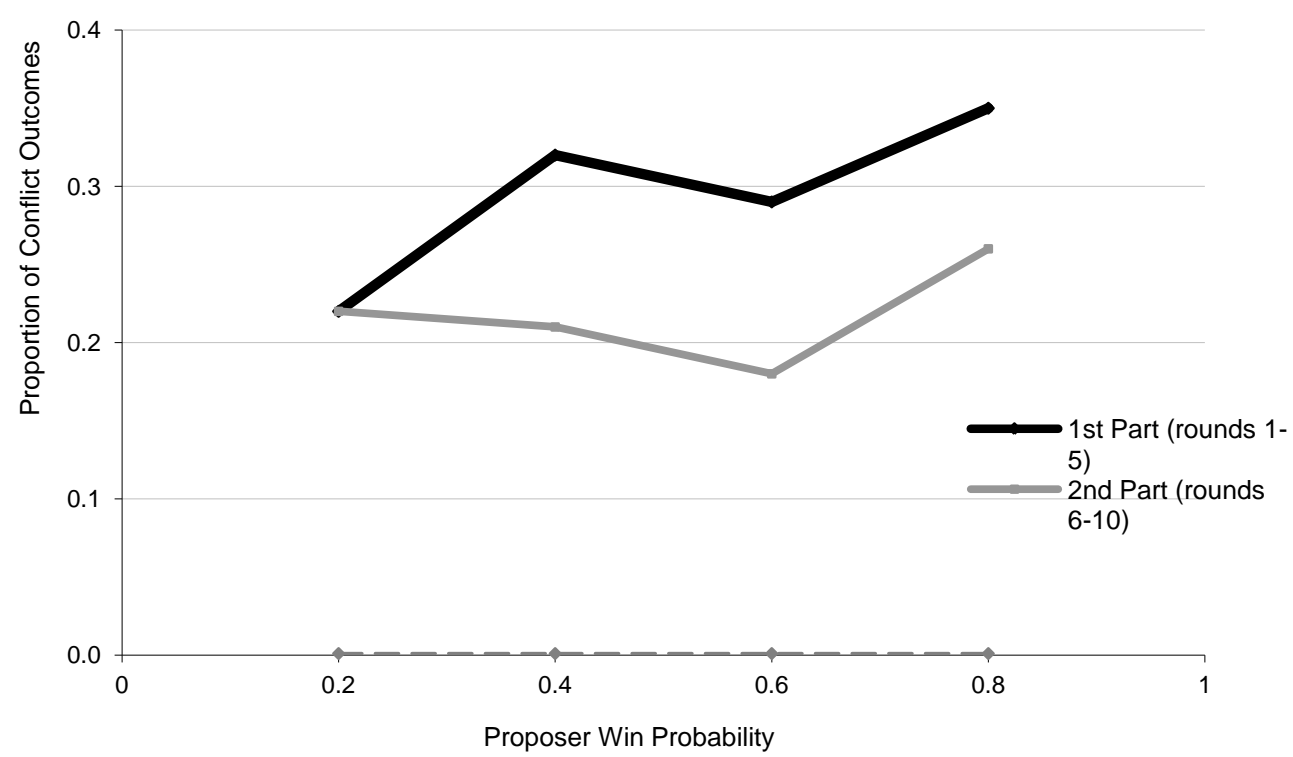


The overall picture that emerges from Figures 1-5 is that proposers with power use it aggressively, with initial demands that typically exceed risk neutral Nash predictions. Responder acceptance proportions, which exhibit regular inverse S-shaped patterns, are also higher than theoretical predictions. The effect of proposer demands driven by power asymmetries is, therefore, mitigated by a countervailing caution on the side of responders. The net effect of these strategic interactions is that conflict rates are significant but are no higher in the presence of strong power asymmetries.

The theoretical predictions are based on an assumption of risk neutrality, and it is likely that attitudes toward risk and fairness play a role, at a minimum, in terms of increasing the variability of behavior across individuals. The overall effect of risk preferences is difficult to ascertain, since the tendency for proposers to make aggressive initial demands and reject counteroffers would be consistent with risk seeking behavior, but the tendency for responders to accept initial demands (more often than would be implied by risk neutrality) could be indicative of risk aversion. One thing is clear, the presence of random elements in the conflict stage, together with heterogeneity in terms of the subjects' feelings about risk and fairness, could have the effect of increasing conflict rates above what would be the case if conflict outcomes were known with certainty. Evidence from ultimatum game experiments with a known conflict outcome ( 0 payoffs for both players) does indicate that conflicts can occur even in the absence of uncertainty, especially when the ultimatum demand is deemed to be unfair. On the other hand, players in disadvantaged positions in multi-stage bargaining games may demand more (something closer to a "fifty-fifty" split), which can also generate conflicts. In the conflict bargaining experiment, subjects may derive some value from a conflict that involves an interesting throw of the dice or that imposes a cost on the other player who rejected one's own "fair" offer. We will first consider whether a standard model based on the player's own experiment payoffs explains observed conflict rates. Then in the estimation section below, we will consider evidence that subjects derive some extra value from conflict.

\subsection{Statistical Tests}

The primary prediction of the model, that the share demanded by the proposer is an increasing function of that person's power (as measured by the win probability) is clearly supported (in a qualitative sense) by a "within subjects" comparison of average initial demands for the first and second parts of each session. In all 12 cases, the average initial demand is higher for the treatment with the higher proposer win probability. This result is significant using a sign test $(p=0.001){ }^{8}$

\footnotetext{
8 The total numbers of accepted and rejected demands for all sessions are presented in the Data Appendix at the end of this paper. Data tables for all sessions are available on the web at: http://www.people.virginia.edu/ cah2k/pcg_data.htm The sessions are numbered pcg1-pcg13, with the omission of pcg6 which was a test conducted at another university for demonstration purposes. For each session, the data table provides links to a graph of data averages by round, along with data summaries in ".htm" and spreadsheet formats for each session. The treatment orders and average initial proposer demands for all sessions are:
}

Session pcg1: $\$ 5.17$ for $\mathrm{P}=0.2, \$ 6.83$ for $\mathrm{P}=0.8$

Session pcg2: $\$ 5.37$ for $\mathrm{P}=0.4, \$ 6.33$ for $\mathrm{P}=0.6$

Session pcg3: $\$ 5.63$ for $\mathrm{P}=0.6, \$ 3.70$ for $\mathrm{P}=0.2$

Session pcg4: $\$ 6.17$ for $\mathrm{P}=0.8, \$ 4.50$ for $\mathrm{P}=0.4$

Session pcg5: $\$ 6.30$ for $\mathrm{P}=0.8, \$ 4.00$ for $\mathrm{P}=0.2$ 
Moreover, a "between-subjects" comparison of initial demand averages by session indicates that the ranking of initial demand averages is roughly consistent with the ranking of proposer win probabilities. These session averages are shown in Table 1 for the two parts (rounds 1-5 and rounds 6-10). For the first part, shown in the left column, there is only one "reversal," i.e., the $\$ 6.30$ average for one of the 0.6 sessions is greater than the $\$ 6.17$ average for one of the 0.8 sessions. In addition, there is one "tie" at $\$ 6.30$. The most extreme data pattern in the hypothesized direction would be to have no reversals, and the nonparametric Jonckheere test essentially provides the probability (under the null hypothesis of no effect) of seeing something at least as extreme as the observed pattern. ${ }^{9}$ For the first part with one reversal and one tie, the test statistic is $\mathrm{J}=51$, and for the second part with a single reversal, the test statistic is $\mathbf{J}=52$. These are significant at $p=0.005$.

Table 1. Average Proposer Initial Demands by Session.

\begin{tabular}{ccc}
\hline Proposer Win Probability & First Part (rounds 1-5) & Second Part (rounds 6-10) \\
\hline 0.2 & $\$ 4.37, \$ 4.83, \$ 5.17$ & $\$ 3.70, \$ 4.00, \$ 4.47$ \\
0.4 & $\$ 5.30, \$ 5.37, \$ 5.50$ & $\$ 4.50, \$ 4.73, \$ 4.87$ \\
0.6 & $\$ 5.53, \$ 5.63, \$ 6.30$ & $\$ 6.17, \$ 6.33, \$ 6.93$ \\
0.8 & $\$ 6.17, \$ 6.30, \$ 7.30$ & $\$ 6.83, \$ 7.10, \$ 7.57$ \\
\hline
\end{tabular}

The average responder demands by session, shown in Table 2 for each part, show the reverse pattern, with lower responder demands as the proposer win probability increases. This correlation is significant at $p=0.005$ using the Jonckheere test $(\mathrm{J}=46$ for part 1 and $\mathrm{J}=52$ for part 2.)

Table 2. Average Responder Counter Demands by Session.

\begin{tabular}{ccc}
\hline Proposer Win Probability & First Part (rounds 1-5) & Second Part (rounds 6-10) \\
\hline 0.2 & $\$ 5.90, \$ 6.64, \$ 7.25$ & $\$ 7.06, \$ 7.07, \$ 7.18$ \\
0.4 & $\$ 5.21, \$ 5.59, \$ 5.82$ & $\$ 6.08, \$ 6.50, \$ 6.57$ \\
0.6 & $\$ 4.50, \$ 5.27, \$ 5.27$ & $\$ 3.83, \$ 4.25, \$ 4.71$ \\
0.8 & $\$ 3.76, \$ 4.78, \$ 5.18$ & $\$ 3.47, \$ 3.76, \$ 4.17$ \\
\hline
\end{tabular}

The story that emerges from these tests is that the power asymmetry treatment has a systematic effect on both proposer and responder demands. These effects could, in principle, be diminished in the 2nd part if the effect of the 1st part treatment tends to persist. This does not seem to be the case for the proposer and responder demand patterns, since the treatment effects in the second part do not appear to exhibit more noise. The qualitative patterns of proposer and responder demands and conflict rates are

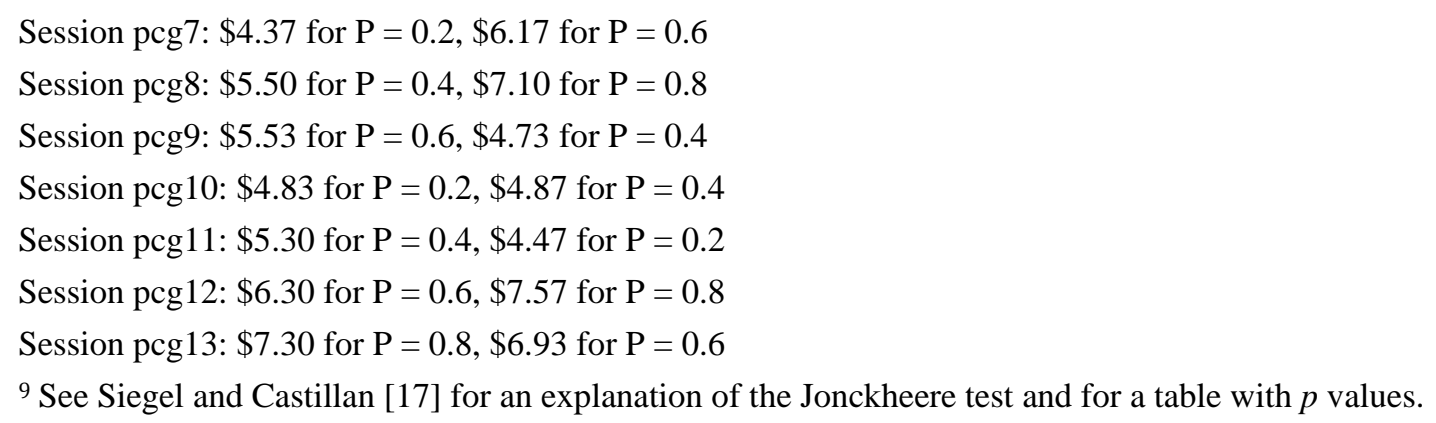


the similar for both the 1st and 2nd parts, and hence, the main treatment effects are not obscured by order effects.

To take a closer look at order effects, consider Table 3, which shows average proposer demands in the 2nd part, organized by 2nd part treatment (left column) and by 1st part treatment (top row). In all columns, an increase in 2nd part proposer power (moving down the column) tends to increase average proposer demands. The effects of increases in 1st part proposer power on 2 nd part demands (moving left to right) are not in the same direction for all rows. This suggests that order effects, if they are present, are subtle.

Table 3. Part 2 Average Proposer Demands for All 12 Sessions, Sorted by Current Power Treatment (Rows) and Previous Power Treatment (Columns).

\begin{tabular}{lcccc}
\hline & 1st Part $P=0.2$ & 1st Part $P=0.4$ & 1st Part $P=0.6$ & 1st Part $P=0.8$ \\
\hline 2nd Part $\mathrm{P}=0.2$ & - & 4.47 & 3.70 & 4.00 \\
2nd Part $\mathrm{P}=0.4$ & 4.87 & - & 4.73 & 4.50 \\
2nd Part $\mathrm{P}=0.6$ & 6.17 & 6.33 & - & 6.93 \\
2nd Part $\mathrm{P}=0.8$ & 6.83 & 7.10 & 7.52 & - \\
\hline
\end{tabular}

One possibility is that order effects are the result of the increased "steepness" in the 2nd part proposer and responder demands, which is apparent from Figures 1 and 4 . If this increased steepness is due to the residual impact of a treatment change in round 6 , then the steepness might be expected to be more enhanced in round 6 than in round 10. This is not the case, since the average proposer demands in round 6 rise from $\$ 4.39$ (for $P=0.2$ ) to $\$ 6.94$ (for $P=0.8$ ), whereas the rise is somewhat sharper in round 10 , from $\$ 3.83$ (for $P=0.2$ ) to $\$ 7.56$ (for $P=0.2$ ). ${ }^{10}$ An alternative explanation is that the enhanced steepness of proposer and responder demands in Figures 1 and 4 is due to more "Nash-like" behavior in later rounds. We will consider this possibility in the estimation section 6 .

Another interesting possibility is that the increases in power asymmetry from parts 1 to 2 may increase conflict rates. Some evidence consistent with this perspective is provided by Table 4, which shows session average conflict rates for the 2 nd part, sorted by current and previous treatment parameters. The four sessions with an increased degree of power asymmetry are marked with an asterisk, e.g., a change from $P=0.4$ (a "forty-sixty" split) in the first part to 0.2 (a "twenty-eighty" split) in the second. The other eight sessions are characterized by a decreased power asymmetry (e.g., from 0.8 to 0.6 ) or no change in asymmetry (e.g., from 0.8 to 0.2 ). The conflict rates for the four sessions with increased power asymmetry are, on average, higher than the conflict rates for the other

\footnotetext{
${ }^{10}$ As a rough descriptive exercise, we ran simple linear regression of 2 nd part average proposer demands by session on 1 st and 2 nd part values of the proposer win probability, $P$. A positive coefficient for the 1 st part value of $P$ would indicate an inertia effect, so that more proposer power in the 1st part shows up as higher demands in the second part. In contrast, a negative coefficient for the 1 st part value of $P$ would tend to make the average demand line for the 2 nd part steeper in Figure 1, as is observed. For example, when $P$ is low in the 2 nd part, the first part treatment tends to be higher, and a negative coefficient on the 1 st part value of $P$ would make proposer demands in the 2 nd part even lower. As expected, the coefficient for the 2 nd part value of $P$ was positive and highly significant $(t=9.6)$, but the coefficient for the 1 st part value of $P$ was positive and insignificant $(t=0.6)$. This analysis suggests that the steepness of average proposer demands for the 2nd part in Figure 1 is not primarily due to order effects.
} 
eight sessions. This difference is statistically significant $(p=0.05)$ using a permutation test (24 of the 495 possible permutations of sessions averages between treatments result in treatment differences as or more extreme than what is observed). This order effect would imply higher conflict rates at the extremes of $P=0.2$ in the top row and at $P=0.8$ in the bottom row, each of which involve an increase in asymmetry in two of three sessions for that row. This could be an explanation for the very slight "U" shape in the conflict rates observed in Figure 5 for the 2nd part. It would be interesting to design an experiment with large changes in power asymmetry, in order to evaluate the effects of these changes on conflict rates.

Table 4. Part 2 Average Conflict Rates for All 12 Sessions, Sorted by Current Power Treatment (Rows) and Previous Power Treatment (Columns).

\begin{tabular}{lllll}
\hline & 1st Part $P=\mathbf{0 . 2}$ & 1st Part $P=\mathbf{0 . 4}$ & 1st Part $P=\mathbf{0 . 6}$ & 1st Part $P=\mathbf{0 . 8}$ \\
\hline 2nd Part $\mathrm{P}=0.2$ & & $\mathbf{0 . 2 3} *$ & $\mathbf{0 . 2 0} *$ & 0.20 \\
2nd Part $\mathrm{P}=0.4$ & 0.33 & & 0.13 & 0.17 \\
2nd Part $\mathrm{P}=0.6$ & 0.13 & 0.20 & & 0.20 \\
2nd Part $\mathrm{P}=0.8$ & 0.13 & $\mathbf{0 . 3 7} *$ & $\mathbf{0 . 3 0} *$ & \\
\hline \multicolumn{5}{r}{ * These are sessions with an increase in power asymmetry in part 2. }
\end{tabular}

\section{Experimental Implications}

The experimental results show some evidence for the imperfect use of subgame perfect Nash equilibrium strategies. Although the demands and counteroffers fail to match game theoretic predictions, they do indicate that subjects involved in a bargaining game with a conflict stage take relative power into account when making demands. Although the qualitative features of some of the Nash predictions are apparent in the data, the systematic directional biases we observe are hardly surprising, given the results of prior laboratory studies (without the conflict stage). ${ }^{11}$ For example, Neelin, Sonnenschein, and Spiegel [13] ran experiments using multi-stage bargaining games. Their results for two-stage games reflect a certain regularity, subjects who are disadvantaged in the sense that they are predicted to demand less than half of the pie tend to be more aggressive, and subjects who are predicted to obtain a large share of the pie tend to demand less than they "should," which is in line with ultimatum game experiments.

Anomalous results in bargaining experiments are typically thought to reflect concerns for fairness, rather than straightforward self-interested strategic behavior [9]. Goeree and Holt [7] exaggerated fairness considerations by providing subjects in two-stage bargaining games with unequal and known outside income payments. These "outside income" payments changed the equal final payoff locus, and exogenously induced inequities seemed to have a more prominent effect than delay cost changes that altered selfish Nash predictions. In many legal and political settings, however, it is hard to imagine that the actors have much concern for others' payoffs. Instead of enhancing equity considerations, it may be instructive to move in the other direction. In particular, equity considerations in the lab can be

\footnotetext{
${ }^{11}$ Failure to see subgame perfect equilibrium results in bargaining experiments has become so frequent that it is the rule, rather than the exception [16,1]. The conflict between theory and experimental results in bargaining games is so prominent that Samuelson [16] highlights this case in describing the role of experiments in testing and improving economic theory.
} 
dampened by providing unequal private incomes that are not common knowledge, which would make equal-payoff outcomes difficult to ascertain. We conjecture that the effect of having a contest at the end of each game in our experiment is to create a competitive atmosphere that dampens concerns for fairness.

The addition of the random conflict lottery can introduce another source of potential bias, since anomalous results for risky choices are quite common. For example, probabilities might be overweighted if they are small. For early evidence supporting the notion of nonlinear probability weighting, see Cohen, Jaffray and Said [3], who report results from an experiment with choices between a sure option and a lottery and observe less sensitivity to probabilities in their subjects' choices than would be expected. In particular, subjects seemed to overweight low probabilities of gains and underweight high probabilities of gains, with the result being a relative insensitivity to intermediate probabilities. Similar patterns are reported by Kahneman and Tversky [11]. Our data are consistent with these results in the sense that an overweighting of low proposer win probabilities might cause them to think that they can demand more and that responders would accept less than would be predicted on the basis of expected value calculations. ${ }^{12}$ Nevertheless, the biases away from risk-neutral Nash predictions in Figures 1, 4, and 5 are not concentrated at the extremes of 0.2 and 0.8 , which suggests another explanation.

Finally, it is well known from "centipede" and other multi-stage games that backward induction does not come easily to subjects. Binmore, Shaked, and Sutton [2] dismiss the notion that we should expect to see subgame-perfect behavior in the laboratory, because even they, as designers, had to work to come up with the game theoretic results for their ultimatum bargaining game. ${ }^{13}$ Even in the absence of the need for backward induction and probability assessments, e.g., a 1-stage bargaining game with no conflict, the Nash predictions are based on a perfect rationality assumption that a gain of only a penny would be enough to induce the other person to accept a final offer. A small amount of irrationality could produce random responses, and the effects of such randomness should be magnified in the presence of multiple stages and the exogenous uncertainty of a conflict. The next section presents a quantal response model of conflict bargaining in which "irrational" rejections or acceptances become more likely as expected payoff differences diminish, which provides an incentive for responders to be cautious with final counter-offers that are too near the "edge." The converse of this is that proposers may be more aggressive with initial demands knowing that responders may accept a smaller share if they anticipate being cautious with counteroffers. By construction, a model with errors will produce conflicts. The interesting question, however, is whether such a model (without inequity aversion and probability misperception) can produce (1) proposer demands that are above Nash levels and too flat; (2) responder demands that are below Nash levels and too flat; and (3) conflict proportions that are significant and relatively invariant to power asymmetries.

\footnotetext{
12 Probability weighting can also explain anomalies like the Allais paradox. See the discussion in Davis and Holt [4].

13 They stress the frequent need for a rule of thumb in understanding problems. However, they indicate that learning and experience can bring players closer to the theoretical predictions. Comparisons of first-half and second half data for proposals and conflicts in Figures 1, 4, and 5 offer some support for this point of view, despite the fact that clear deviations from subgame-perfect Nash predictions do persist.
} 


\section{Estimation of a Quantal Response Model of Two-Stage Bargaining}

Consider a proposer who receives a counter offer of $9-x_{2}$ in the final stage and must decide whether to accept or reject. A conflict with a win probability of $p$ yields an expected payoff of $9 p-c_{p}$. Given a logit precision parameter of $\lambda$, the probability that the counteroffer is accepted is calculated as a ratio of exponentials of precision-adjusted expected payoffs for accepting and rejecting:

$$
\operatorname{Pr}\left(\text { accept } \mid x_{2}, p\right)=\frac{\exp \left(\lambda\left(9-x_{2}\right)\right)}{\exp \left(\lambda\left(9-x_{2}\right)\right)+\exp \left(\lambda\left(9 p-c_{p}\right)\right)},
$$

which converges to 0.5 as $\lambda \rightarrow 0$ (pure noise) and which converges to 1 only if the payoff for accepting, $9-x_{2}$, is higher than the expected conflict payoff (perfect rationality). Also, note that equal expected payoffs result in choice probabilities of $1 / 2$ in this case. ${ }^{14}$

Given $\lambda$, which is assumed to be the same for the responder, the model is solved by backward induction by letting the responder probabilities for each demand $x$ be determined analogously as ratios of precision-adjusted expected payoffs. The responder's payoff for making a demand of $x_{2}$ depends on the acceptance probability from Equation (2) and the expected conflict payoff: $\operatorname{Pr}\left(\text { accept } \mid x_{2}, p\right)^{*} x_{2}+\left(1-\operatorname{Pr}\left(\text { accept } \mid x_{2}, p\right)\right)^{*}\left(9(1-p)-c_{r}\right)$. Let this expected payoff for a responder counter demand of $x_{2}$ be denoted by $\pi\left(x_{2} \mid p\right)$. In a quantal response equilibrium (McKelvey and Palfrey, 1995), the assumption is that the proposer choice probability in Equation (2) is equal to the responder's belief probability used to construct the expected payoff for each possible responder counter-offer. Then the responder's choice probabilities for each possible counter-demand are calculated:

$$
\operatorname{Pr}\left(\text { demand } x_{2} \mid p\right)=\frac{\exp \left(\lambda \pi\left(x_{2} \mid p\right)\right)}{\sum_{i=1,9} \lambda \pi(i \mid p)}
$$

for $x_{2}=1, \ldots 9$, where the denominator ensures that the choice probabilities for each of the 9 possible counter demands sum to 1 . As before, a very high value of the precision parameter will put probability 1 on the expected-payoff-maximizing demand.

Proposer demands and acceptance probabilities for the first stage are calculated analogously by computing a "value" for optimal continuation for each player. The value of continuation would be the proposer's expected payoff associated with a vector of possible responses from Equation (3) and an assumption that the proposer's final decisions about whether to accept or reject will be in accordance with the acceptance probabilities from Equation (2). ${ }^{15}$ In this manner, the single precision parameter

\footnotetext{
14 The logit probabilistic choice function is derived by assuming that the random errors associated with each expected payoff have a particular (double exponential) distribution. The implied assumption that these errors are identically and independently distributed is needed to avoid a model in which any distribution of choice probabilities can be achieved by manipulating the error distributions for each payoff term (see Goeree, Palfrey, and Holt, 2006, for examples of such manipulations). In other words, the assumption of independent and identically distributed errors is needed to ensure that the probabilistic choice model actually has empirical content, regardless of whether the model involves isolated individual decisions in a standard logit analysis or strategic interactions in a quantal response equilibrium. To reiterate, it is a common misunderstanding to believe that any pattern of choice proportions can be explained with a quantal response model, but this observation is only true for a general error specification that does not require "i.i.d." error terms.

15 Notice that this is a "quantal continuation value," not an optimal continuation value (which would involve always making the decision that provides the highest expected payoff).
} 
enters all of the various acceptance and rejection probabilities and demand probabilities for proposers and responders. Estimation involves finding the value of $\lambda$ that maximizes the probability of seeing the data pattern that was actually observed. The likelihood function is a product of terms, each involving a probability for a particular category raised to a power representing the number of observations in that category. The data consist of the numbers of accepted and rejected demands for each of the four proposer win probabilities $(0.2,0.4,0.6,0.8)$ for each of the 10 possible initial demands and each of the 9 possible counter-offers.

This model is a one-parameter generalization of the Nash equilibrium determined by $\lambda$, which for each value of $p$ generates 9 proposer acceptance probabilities from Equation (2), 10 responder acceptance probabilities for each value of $p$, and the probabilities associated with each possible initial proposer demand and each possible responder counter offer. All of these probabilities are functions of the proposer win probability $p$. Using data from the second half (rounds 6-10), we obtained a maximum likelihood estimate of $\lambda=1.110$, with a standard error of $0.031{ }^{16}$ The maximized likelihood function was: $-2,857$. The vectors of initial proposal probabilities were used to calculate QRE predictions for average proposer demands for each proposer win probability, as shown by the dark dot-dash line in Figure 6. As is the case for the second half data averages (gray line), these QRE predictions lie above the Nash prediction and are too flat relative to the Nash prediction. The proposers in the experiment, however, are making demands that are not quite as high as predicted by the QRE estimates.

Figure 6. Average Proposer Demands (Second Part) and QRE Predictions.

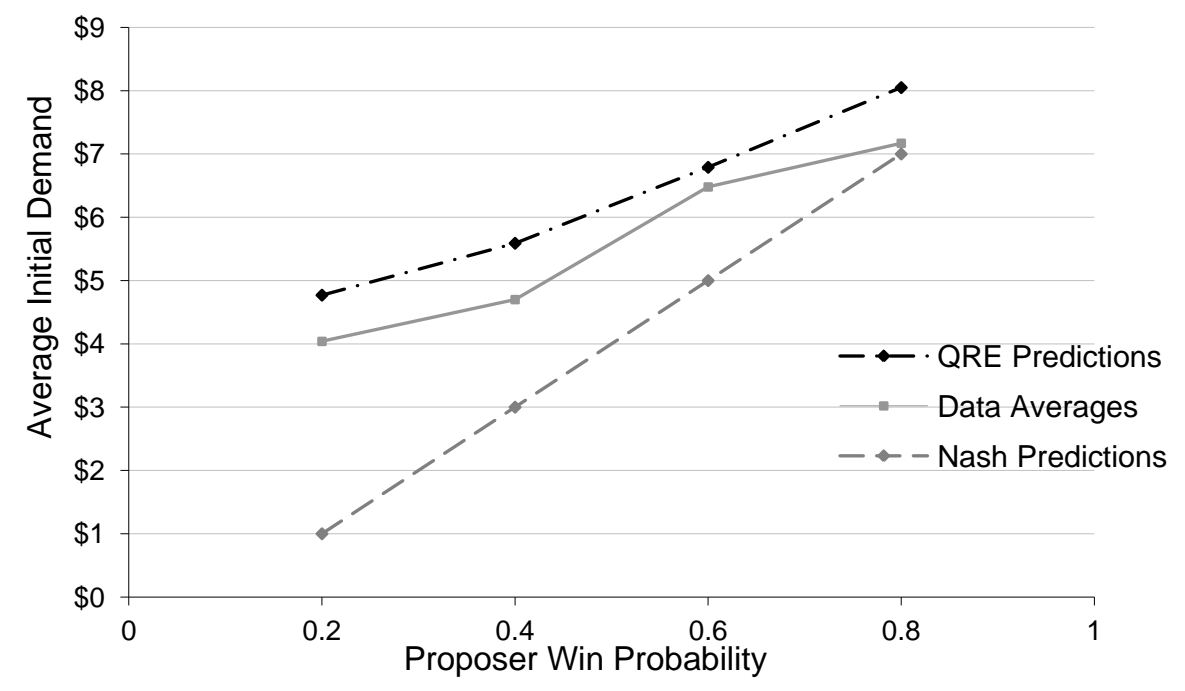

Similarly, the QRE predictions for the responder demands trace out a line that begins below the dashed Nash prediction line in Figure 7 and is too flat relative to the that line. This feature of the QRE predictions matches the second half data (gray line).

Finally, note that the QRE predictions for conflict rates shown in Figure 8 are at about the level observed in the second-half data, but without the upward spike for the highest proposer win

\footnotetext{
${ }^{16}$ Estimation based on the first half data provides a lower precision, $\lambda=0.762$, which implies more "noise," as would be expected.
} 
probability. These predictions are a major improvement over the Nash prediction of zero conflict rates for all power treatments.

Figure 7. Average Responder Demands (Second Part) and QRE Predictions.

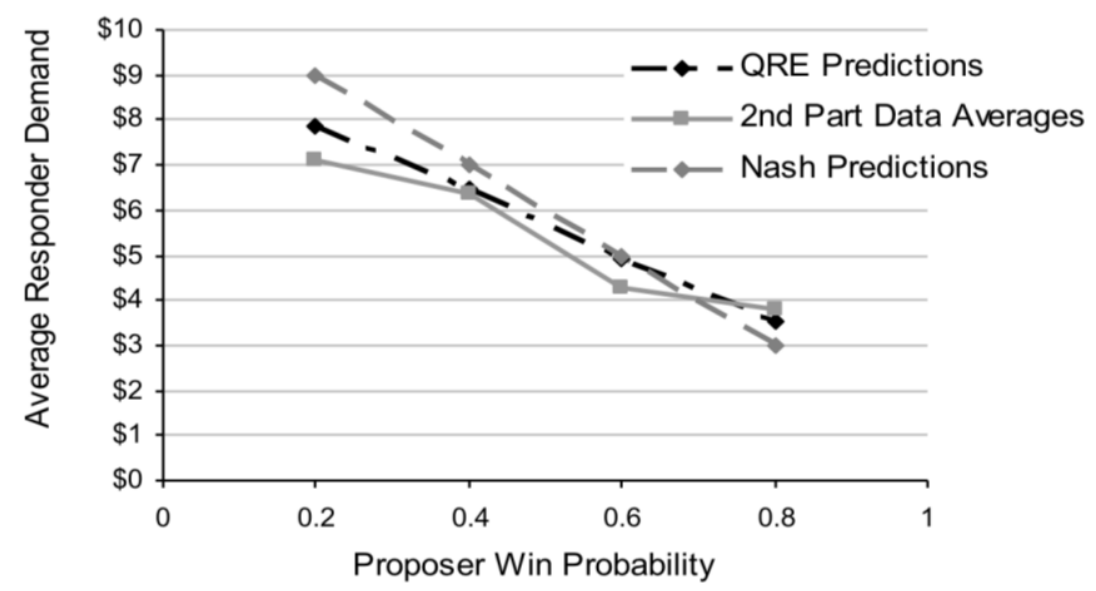

Figure 8. Conflict Rates (Second Part) and QRE Predictions.

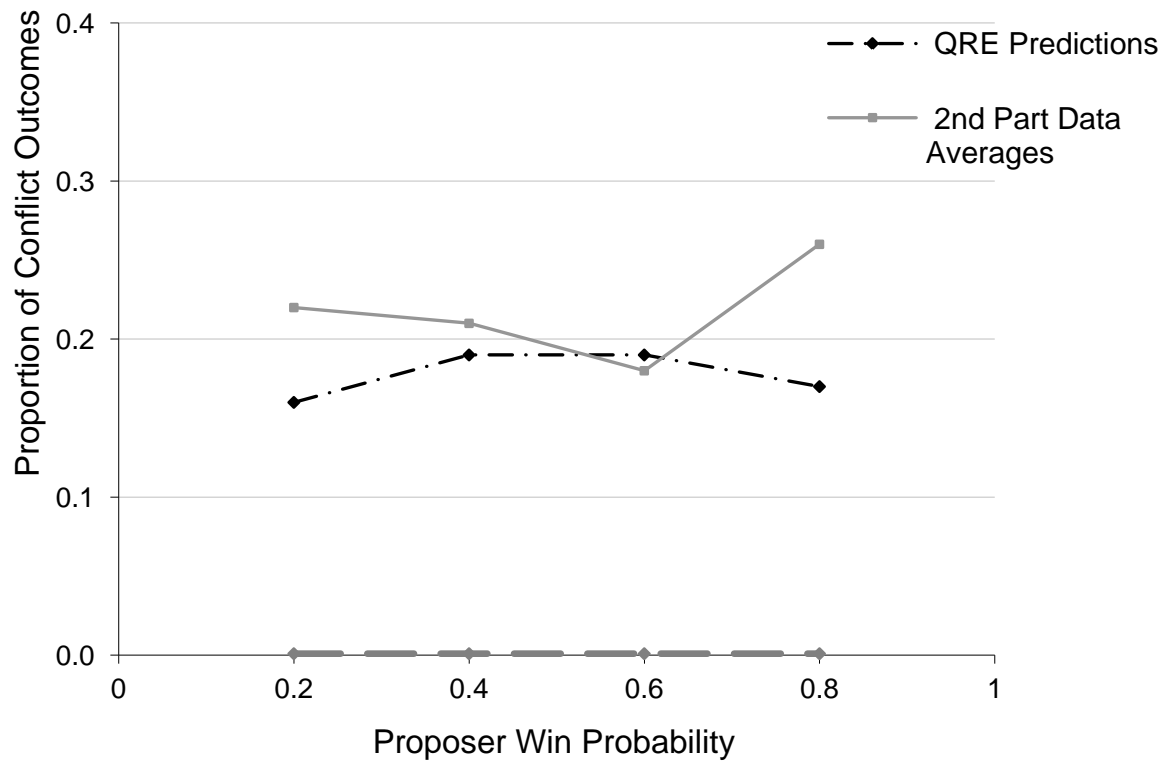

An analysis of the proposer "continuation values" provides an interesting insight as to why initial proposer demands might be too high and too flat relative to Nash predictions. The proposer continuation values implied by the QRE choice probabilities are shown in the top row of Table 3. For example, a proposer with a win probability of 0.2 (and who has an initial demand rejected) can expect to earn $\$ 0.99$ in the final stage, largely because responders are less aggressive than predicted in this stage in anticipation of "irrational" rejections. The Nash continuation value after a rejected initial proposal would be 0 for this proposer, since the 0.2 chance of winning $\$ 9$ in a conflict is less than the $\$ 2$ conflict cost, so a responder second-stage counter-offer of $\$ 0$ "should" be accepted by a perfectly rational proposer. The other proposer continuation values in the top row are also greater than the Nash continuation values in the second row, although the difference goes away for the highest proposer win probability of 0.8 . The important point is that the higher the proposer's continuation value from 
rejection, the higher the optimal initial demand, which explains why proposer demands lie above Nash levels for win probabilities below 0.8 .

The bottom part of Table 5 also shows the inferred continuation values for responders following their rejection of an initial proposer demand. For example, with a proposer win probability of 0.2 , the responder continuation value is $\$ 9$ since the proposer has no power when the conflict cost exceeds the proposer's expected gain from conflict. Note that the inferred responder QRE continuation values in the third row are below their Nash continuation values in the bottom row, presumably because of the possibility that proposers will sometimes reject aggressive responder demands and force the responder into a costly conflict. This possibility reduces the optimal responder demand, which helps explain the qualitative direction of deviations from Nash predictions in Figure 7.

Table 5. Continuation Values Inferred From Quantal Response (QRE) and Nash Models.

\begin{tabular}{ccccc}
\hline Proposer Win Probabilities: & $\boldsymbol{p}=\mathbf{0 . 2}$ & $\boldsymbol{p}=\mathbf{0 . 4}$ & $\boldsymbol{p}=\mathbf{0 . 6}$ & $\boldsymbol{p}=\mathbf{0 . 8}$ \\
\hline Proposer Continuation Values (QRE): & $\$ 0.99$ & $\$ 2.54$ & $\$ 4.25$ & $\$ 5.89$ \\
Proposer Continuation Values (Nash) & $\$ 0$ & $\$ 2$ & $\$ 4$ & $\$ 6$ \\
\hline Responder Continuation Values (QRE) & $\$ 7.05$ & $\$ 5.15$ & $\$ 3.28$ & $\$ 1.47$ \\
Responder Continuation Values (Nash) & $\$ 9$ & $\$ 7$ & $\$ 5$ & $\$ 3$ \\
\hline
\end{tabular}

One unresolved issue that came up in the earlier discussion of experimental results is the observation that both proposer and responder demand patterns are "flatter" (less responsive to proposer win probabilities) in the first five rounds than was the case in the last five rounds. We do not believe that this difference was caused by the treatment change, as discussed previously, since the steepness of demand patterns continued to increase from round 6 to round 10. /This observation suggests some kind of learning or other change in attitudes. Note that the steeper demand lines for part 2 in Figure 1 (for proposers) and Figure 4 (for responders) are closer to the dotted line Nash predictions, which suggests an increase in the precision parameter $\lambda$. Estimation of the QRE model using only data from the first five rounds yields an estimate of $\lambda=0.76$ (standard error $=0.025$ ), which is in fact lower than the estimate of $\lambda=1.11$ (standard error $=0.031$ ) using data from the final five rounds. These different parameter estimates can be used to generate "predictions" for the patterns of average proposer and responder demands for each part. The lower precision estimate for part 1 (more noise) does yield a flatter predicted response of proposer and responder demands to changes in the proposer win probability, $P$, as shown in Table 6 . The top part of the table indicates that predicted proposer demands are less responsive to increases in $P$ in part 1 than in part 2. The bottom half of the table shows a similar pattern for responder demands (more flatness in predictions for the first part). In terms of levels and crossover points, these predictions do deviate somewhat from the actual data patterns shown in Figures 1 and 4, but the general qualitative differences in relative flatness are present in the QRE predictions. 
Table 6. Quantal Response Predictions for Proposer and Responder Demands Using Separate Precision Estimates for Part 1 (Rounds 1-5) and Part 2 (Rounds 6-10).

\begin{tabular}{ccccc}
\hline & $\boldsymbol{P}=\mathbf{0 . 2}$ & $\boldsymbol{P}=\mathbf{0 . 4}$ & $\boldsymbol{P}=\mathbf{0 . 6}$ & $\boldsymbol{P}=\mathbf{0 . 8}$ \\
\hline Predicted Proposer Demands, Part 1 & $\$ 5.19$ & $\$ 5.87$ & $\$ 6.90$ & $\$ 7.92$ \\
Predicted Proposer Demands, Part 2 & $\$ 4.77$ & $\$ 5.59$ & $\$ 6.80$ & $\$ 8.05$ \\
\hline Predicted Responder Demands, Part 1 & $\$ 7.50$ & $\$ 6.47$ & $\$ 5.24$ & $\$ 4.14$ \\
Predicted Responder Demands, Part 2 & $\$ 7.85$ & $\$ 6.46$ & $\$ 4.92$ & $\$ 3.51$ \\
\hline
\end{tabular}

To summarize, maximum likelihood estimation of a simple model that adds a "noise" dimension to the standard game-theoretic analysis provides a logit precision parameter that, in turn, generates predicted decision patterns, which are similar to the patterns observed in the data:

(1) Proposer initial demands are increasing in proposer win probabilities, as expected, but are above Nash levels and are "too flat,"

(2) Responder final demands are decreasing in proposer win probabilities, but are below Nash levels and are also too flat.

(3) Conflict rates are significant and are roughly invariant to changes in proposer win probabilities.

Separate estimation for the part 1 and part 2 data suggests that noise declines in the final five rounds, which explains the reduced flatness in the proposer and responder demand patterns, and the reduction in conflict rates observed in the second part.

One interesting divergence between data and predictions of this one-parameter model is that conflict rates are under-predicted for both parts. In the first part, the predicted conflict rate (averaged over all four proposer win probabilities) is 0.21 , whereas the observed conflict rate is close to 0.30 . In the second part, the predicted conflict rate was 0.18 , again below, but only slightly below, the observed rate of 0.22 . As discussed earlier, it could be the case that subjects in the experiments derive some extra value from conflict, e.g., if there is some excitement in seeing a random outcome, or if there is a feeling of satisfaction from imposing a conflict cost on the other player after one's offer has been rejected. ${ }^{17}$ As a result, we do not think too much importance should be attached to the overall levels of observed conflict rates. An admittedly ad hoc inclusion of a "conflict value" term in both proposer and responder conflict payoffs yields estimated values of about a dollar for each part. ${ }^{18}$

\footnotetext{
${ }^{17}$ The possible interest in seeing a random outcome instead of a predictable payoff was suggested to us by an anonymous referee. A second referee suggested that risk aversion and fairness considerations may enhance the desirability of conflict.

${ }^{18}$ The estimated conflict values and standard errors are \$1.08 (0.11) for part 1 and \$0.74 (0.08) for part 2, which indicates a moderate preference for conflict. This added parameter has little effect on the estimates of the logit precision parameter, the maximized values of the log likelihood, and the resulting patterns of predicted proposer and responder demands and the flat profile of conflict rates. It is no surprise that the inclusion of a value of conflict does raise predicted conflict rates, from 0.21 to 0.32 in the first part, which is close to the observed average of 0.30 , and from 0.18 to 0.24 for the second part, which is also close to the observed average of 0.22 in the second part.
} 


\section{Conclusion}

This paper examines how the probability of winning in a conflict shapes the demands and counter-demands that are made in initial stages of the bargaining process. The experimental setup is novel in that it allows us to examine an important issue in the literature of international relations, i.e., how conflict rates are affected by power asymmetries that would come into play in the event of an impasse. The main results are intuitive, that demands and counter-demands are sensitive to relative power should a conflict arise, and that conflict does occur in a significant proportion of cases. Conflict rates seem to be unaffected by power asymmetries, since the players adjust their demands based on the asymmetries. What is observed is that proposers with power use their power to make aggressive demands. However, the aggressive demands by the proposers are received by responders who react with countervailing caution.

It is impractical to run experiments with real conflicts between political decision makers. We believe that the use of experimental methods provide a venue for testing expectations about bargaining and conflict and helps avoid problems of measurement and control that arise with field data, although there is clearly a cost in terms of external validity. ${ }^{19}$ Our results are interesting in many ways given the prominence of the bargaining model in the recent literature on international conflict. Although the actual demands do not match the subgame-perfect Nash predictions, the results are qualitatively consistent with expectations from bargaining theory. Conflict outcomes are reduced but remain significant with repeated play. The implications of these results are interesting to consider. It appears that when subjects bargain, they are influenced by the structure of the game, the basic comparative-statics results derived from a Nash equilibrium are observed in the data and supported by appropriate nonparametric tests.

There are, however, systematic discrepancies between observed demands and subgame-perfect Nash predictions. These discrepancies could be a result of difficulties with backward induction reasoning that may inject behavioral "noise" into decision making. Probabilistic choice functions are typically used to relax the standard assumption of perfect rationality. This approach is especially helpful for the analysis of experimental data that show "smoothed" responses, as can be seen from the "inverse S" shapes of responder accept proportions in Figure 3. The quantal response model imposes a fixed-point equilibrium condition that choice distributions match belief distributions; this approach is a simple generalization of Nash requirement that equilibrium strategies be best responses to other players' strategies. For our experiment, the basic quantal response model provides a simple explanation of the overall pattern of deviations from Nash predictions, without the need to introduce preference parameters that pick up aversions to inequity or risk, although such additions might be useful in other contexts. Note that the introduction of additional unobserved parameters would necessitate estimation. Any estimation requires an error structure, which is precisely what a quantal response model provides, in an equilibrium setting that is appropriate for game theory. Moreover, an analysis of the continuation values inferred from the estimated logit precision parameter provides insights about why proposer demands are above Nash predictions, why responder demands are below

\footnotetext{
${ }^{19}$ This work could be complemented with field studies done with military officers or legal negotiators through a survey design (Tomz and Weeks, forthcoming). Frechette (forthcoming) reviews a series of parallel studies that use both student subjects and relevant professionals. Although there are some differences, student subjects do not exhibit greater deviations from theoretical predictions, as compared with professionals.
} 
Nash predictions, and why both demand patterns are less responsive to power asymmetries than predicted.

Given that risky conflict is a potential outcome of most bargaining procedures, especially those found in political and legal interactions, we believe that additional experimental investigation of this area could provide valuable insights that could later be validated with field experiments. For example, it is likely that the difficulties associated with backward induction can be reduced in a single-stage model or enhanced in games with more stages. Another interesting direction would be to explore the effects of ambiguity, i.e., a situation in which the proposer win probabilities are not known, which is clearly a feature of most field applications. Subjects tend to exhibit more risk aversion in cases of ambiguity [3], which can be implemented by not revealing the numbers of various colored marbles in urns used for random draws. Finally, the alternating-offer structure of these bargaining games could be generalized to allow endogenously or randomly determined decision sequences.

\section{Acknowledgments}

We wish to thank Michael Patashnik for research assistance. This research was funded in part by NSF/NSCC grants 0904795 and 0904798, and NSF/BCS 0904946.

\section{Conflict of Interest}

The authors declare no conflict of interest.

\section{Instructions Appendix}

- Rounds and Matchings: The experiment consists of a number of rounds. Note: In each round, you will be matched with another person selected at random from the other participants. There will be a new random re-matching each round.

- Initial Allocation: The initial status quo allocation involves a payment of $\mathbf{\$ 3 . 0 0}$ to one person, designated as Proposer (initial decision-maker), and an amount $\mathbf{\$ 7 . 0 0}$ to the other person, the responder.

- Initial Decisions: The proposer begins by proposing an allocation of the total, $\mathbf{\$ 1 0 . 0 0}$, that may or may not differ from the initial allocation. The responder must either accept or reject this proposal.

- Second Stage Decisions: If the responder (second-mover) rejects, then the amount available is reduced to $\mathbf{\$ 9 . 0 0}$, and the responder can make a proposal for how to allocate this lower amount. If a counter proposal is made, the initial proposer (first mover), must either accept it or not.

- Dispute Outcome: If the counter proposal is rejected, the resulting dispute is resolved randomly, as explained on the next page.

- Conflict Costs: If the process reaches the dispute stage, then a conflict cost is deducted from earnings; this cost is $\mathbf{\$ 2 . 0 0}$ for the proposer and $\mathbf{\$ 2 . 0 0}$ for the responder, irrespective of the outcome of the dispute.

- Role: You have been randomly assigned to be a Proposer (or initial decision-maker) in this process, and you will begin by suggesting amounts of money for each of you that sum to $\mathbf{\$ 1 0 . 0 0}$. 
- OR Role: You have been randomly assigned to be a Responder in this process. The other person (proposer) will begin by suggesting amounts of money for each of you that sum to $\$ \mathbf{1 0 . 0 0}$.

- Earnings with No Dispute: If the responder accepts the proposed reallocation, then it is implemented. If the responder rejects, then the amount available becomes $\mathbf{\$ 9 . 0 0}$, and the responder must make a counter-proposal on how to allocate this new amount. If the initial proposer accepts this counter-proposal, then it is implemented. If the counter-proposal is rejected, then the process goes to the dispute stage.

- Earnings with a Dispute: The outcome is determined by the simulated throw of a 10 -sided die, labeled $1,2, \ldots 9,10$, where each outcome is equally likely. If the throw is less than or equal to 2 , then the proposer wins, so the chances that the proposer wins are 2 out of 10 , which translates into a probability .2 that the proposer wins. The winner of the dispute obtains the full amount available at this stage \$9.00, minus their conflict cost, and the loser receives nothing (a loss equal to the conflict cost, which is $\mathbf{\$ 2 . 0 0}$ for the proposer and $\mathbf{\$ 2 . 0 0}$ for the responder).

- At the beginning of each round, there will be a new random pairing of all participants, so the person who you are matched with in one round may not be the same person you are matched with in the subsequent round. Matchings are random, and you are no more likely to be matched with one person than with another.

- The initial status quo allocation involves a payment of $\mathbf{\$ 3 . 0 0}$ to the proposer and $\mathbf{\$ 7 . 0 0}$ to the responder.

- The proposer begins by suggesting an alternative allocation of the available $\mathbf{\$ 1 0 . 0 0}$; this proposal determines earnings for the round if it is accepted.

- If the initial proposal is rejected, the amount of money available is changed to $\mathbf{\$ 9 . 0 0}$, and the responder must then make a counter-proposal for how to allocate this new amount.

- Finally, the initial proposer must either accept the counter-proposal or reject. An acceptance implements the counter-proposal, and a rejection leads to a conflict.

- In a conflict, the available amount, $\mathbf{\$ 9 . 0 0}$ goes to the proposer with probability.2, and it goes to the responder with probability $\mathbf{. 8}$, as determined by a random process that simulates the throw of a ten-sided die.

- In the event of a conflict, the proposer incurs a cost of $\mathbf{\$ 2 . 0 0}$ and the responder incurs a cost of \$2.00, regardless of the conflict outcome.

- Special Earnings Announcement: Your cash earnings in this experiment will be 50\% of your cumulative earnings at the end of the experiment. 
Data Appendix, First Half (Rounds 1-15)

\begin{tabular}{|c|c|c|c|c|c|c|c|c|c|c|}
\hline Proposer Demands & $\$ 1$ & $\$ 2$ & $\$ 3$ & $\$ 4$ & $\$ 5$ & $\$ 6$ & $\$ 7$ & $\$ 8$ & $\$ 9$ & $\$ 10$ \\
\hline number accepted, $p=0.2$ & $\mathbf{0}$ & $\mathbf{0}$ & 2 & 46 & 32 & $\mathbf{0}$ & $\mathbf{0}$ & $\mathbf{0}$ & $\mathbf{0}$ & $\mathbf{0}$ \\
\hline number rejected, $p=0.2$ & $\mathbf{0}$ & $\mathbf{0}$ & $\mathbf{0}$ & 22 & 60 & 10 & 4 & $\mathbf{0}$ & 4 & $\mathbf{0}$ \\
\hline number accepted, $p=0.4$ & $\mathbf{0}$ & $\mathbf{0}$ & $\mathbf{0}$ & 6 & 84 & 8 & $\mathbf{0}$ & $\mathbf{0}$ & $\mathbf{0}$ & $\mathbf{0}$ \\
\hline number rejected, $p=0.4$ & $\mathbf{0}$ & $\mathbf{0}$ & $\mathbf{0}$ & 4 & 22 & 44 & 8 & 4 & $\mathbf{0}$ & $\mathbf{0}$ \\
\hline number accepted, $p=0.6$ & $\mathbf{0}$ & $\mathbf{0}$ & $\mathbf{0}$ & 2 & 66 & 38 & 2 & $\mathbf{0}$ & $\mathbf{0}$ & $\mathbf{0}$ \\
\hline number rejected, $p=0.6$ & $\mathbf{0}$ & $\mathbf{0}$ & $\mathbf{0}$ & $\mathbf{0}$ & 4 & 34 & 28 & 6 & $\mathbf{0}$ & $\mathbf{0}$ \\
\hline number accepted, $p=0.8$ & $\mathbf{0}$ & $\mathbf{0}$ & $\mathbf{0}$ & $\mathbf{0}$ & 14 & 54 & 42 & $\mathbf{0}$ & $\mathbf{0}$ & $\mathbf{0}$ \\
\hline number rejected, $p=0.8$ & $\mathbf{0}$ & $\mathbf{0}$ & $\mathbf{0}$ & $\mathbf{0}$ & 6 & 12 & 26 & 22 & 2 & 2 \\
\hline Responder Demands & $\$ 1$ & $\$ 2$ & $\$ 3$ & $\$ 4$ & $\$ 5$ & $\$ 6$ & $\$ 7$ & $\$ 8$ & $\$ 9$ & NA \\
\hline number accepted, $p=0.2$ & $\mathbf{0}$ & 2 & 2 & 2 & 8 & 20 & 26 & $\mathbf{0}$ & $\mathbf{0}$ & $*$ \\
\hline number rejected, $p=0.2$ & $\mathbf{0}$ & $\mathbf{0}$ & $\mathbf{0}$ & $\mathbf{0}$ & $\mathbf{0}$ & 6 & 18 & 12 & 4 & $*$ \\
\hline number accepted, $p=0.4$ & $\mathbf{0}$ & $\mathbf{0}$ & $\mathbf{0}$ & $\mathbf{0}$ & 22 & 2 & $\mathbf{0}$ & $\mathbf{0}$ & $\mathbf{0}$ & $*$ \\
\hline number rejected, $p=0.4$ & $\mathbf{0}$ & $\mathbf{0}$ & $\mathbf{0}$ & $\mathbf{0}$ & 22 & 30 & 6 & $\mathbf{0}$ & $\mathbf{0}$ & $*$ \\
\hline number accepted, $p=0.6$ & $\mathbf{0}$ & $\mathbf{0}$ & $\mathbf{0}$ & 8 & 12 & $\mathbf{0}$ & $\mathbf{0}$ & $\mathbf{0}$ & $\mathbf{0}$ & $*$ \\
\hline number rejected, $p=0.6$ & $\mathbf{0}$ & $\mathbf{0}$ & $\mathbf{0}$ & 16 & 18 & 16 & 2 & $\mathbf{0}$ & $\mathbf{0}$ & $*$ \\
\hline number accepted, $p=0.8$ & $\mathbf{0}$ & 2 & $\mathbf{0}$ & 4 & $\mathbf{0}$ & $\mathbf{0}$ & $\mathbf{0}$ & $\mathbf{0}$ & $\mathbf{0}$ & $*$ \\
\hline number rejected,$p=0.8$ & $\mathbf{0}$ & $\mathbf{0}$ & 8 & 30 & 14 & 6 & 6 & $\mathbf{0}$ & $\mathbf{0}$ & $*$ \\
\hline
\end{tabular}

Data Appendix, Second Half (Rounds 6-10)

\begin{tabular}{ccccccccccc}
\hline Proposer Demands & $\$ 1$ & $\$ 2$ & $\$ 3$ & $\$ 4$ & $\$ 5$ & $\$ 6$ & $\$ 7$ & $\$ 8$ & $\$ 9$ & $\$ 10$ \\
\hline number accepted, $\boldsymbol{p}=\mathbf{0 . 2}$ & $\mathbf{0}$ & $\mathbf{1 0}$ & $\mathbf{4 0}$ & $\mathbf{3 2}$ & $\mathbf{1 4}$ & $\mathbf{0}$ & $\mathbf{0}$ & $\mathbf{0}$ & $\mathbf{0}$ & $\mathbf{0}$ \\
number rejected, $\boldsymbol{p}=\mathbf{0 . 2}$ & $\mathbf{0}$ & $\mathbf{0}$ & $\mathbf{2}$ & $\mathbf{3 6}$ & $\mathbf{3 6}$ & $\mathbf{8}$ & $\mathbf{2}$ & $\mathbf{0}$ & $\mathbf{0}$ & $\mathbf{0}$ \\
number accepted, $\boldsymbol{p}=\mathbf{0 . 4}$ & $\mathbf{0}$ & $\mathbf{0}$ & $\mathbf{4}$ & $\mathbf{5 2}$ & $\mathbf{7 0}$ & $\mathbf{2}$ & $\mathbf{0}$ & $\mathbf{0}$ & $\mathbf{0}$ & $\mathbf{0}$ \\
number rejected, $\boldsymbol{p}=\mathbf{0 . 4}$ & $\mathbf{0}$ & $\mathbf{0}$ & $\mathbf{0}$ & $\mathbf{4}$ & $\mathbf{4 0}$ & $\mathbf{8}$ & $\mathbf{0}$ & $\mathbf{0}$ & $\mathbf{0}$ & $\mathbf{0}$ \\
number accepted, $\boldsymbol{p}=\mathbf{0 . 6}$ & $\mathbf{0}$ & $\mathbf{0}$ & $\mathbf{0}$ & $\mathbf{0}$ & $\mathbf{2 4}$ & $\mathbf{9 4}$ & $\mathbf{1 0}$ & $\mathbf{0}$ & $\mathbf{0}$ & $\mathbf{0}$ \\
number rejected, $\boldsymbol{p}=\mathbf{0 . 6}$ & $\mathbf{0}$ & $\mathbf{0}$ & $\mathbf{0}$ & $\mathbf{0}$ & $\mathbf{0}$ & $\mathbf{6}$ & $\mathbf{2 2}$ & $\mathbf{8}$ & $\mathbf{2}$ & $\mathbf{1 4}$ \\
number accepted, $\boldsymbol{p}=\mathbf{0 . 8}$ & $\mathbf{0}$ & $\mathbf{0}$ & $\mathbf{0}$ & $\mathbf{0}$ & $\mathbf{1 8}$ & $\mathbf{1 2}$ & $\mathbf{5 4}$ & $\mathbf{1 0}$ & $\mathbf{0}$ & $\mathbf{0}$ \\
number rejected, $\boldsymbol{p}=\mathbf{0 . 8}$ & $\mathbf{0}$ & $\mathbf{0}$ & $\mathbf{0}$ & $\mathbf{0}$ & $\mathbf{0}$ & $\mathbf{4}$ & $\mathbf{3 2}$ & $\mathbf{3 4}$ & $\mathbf{1 0}$ & $\mathbf{6}$ \\
\hline Responder Demands & $\mathbf{\$ 1}$ & $\mathbf{\$ 2}$ & $\mathbf{\$ 3}$ & $\mathbf{\$ 4}$ & $\mathbf{\$ 5}$ & $\mathbf{\$ 6}$ & $\mathbf{\$ 7}$ & $\mathbf{\$ 8}$ & $\mathbf{\$ 9}$ & $\mathbf{N A}$ \\
\hline number accepted, $\boldsymbol{p}=\mathbf{0 . 2}$ & $\mathbf{0}$ & $\mathbf{0}$ & $\mathbf{0}$ & $\mathbf{0}$ & $\mathbf{0}$ & $\mathbf{1 6}$ & $\mathbf{2 8}$ & $\mathbf{0}$ & $\mathbf{0}$ & $*$ \\
number rejected, $\boldsymbol{p}=\mathbf{0 . 2}$ & $\mathbf{0}$ & $\mathbf{0}$ & $\mathbf{0}$ & $\mathbf{0}$ & $\mathbf{0}$ & $\mathbf{6}$ & $\mathbf{1 6}$ & $\mathbf{8}$ & $\mathbf{1 0}$ & $*$ \\
number accepted, $\boldsymbol{p}=\mathbf{0 . 4}$ & $\mathbf{0}$ & $\mathbf{0}$ & $\mathbf{0}$ & $\mathbf{0}$ & $\mathbf{2}$ & $\mathbf{1 2}$ & $\mathbf{0}$ & $\mathbf{0}$ & $\mathbf{0}$ & $*$ \\
number rejected, $\boldsymbol{p}=\mathbf{0 . 4}$ & $\mathbf{0}$ & $\mathbf{0}$ & $\mathbf{0}$ & $\mathbf{0}$ & $\mathbf{0}$ & $\mathbf{2 4}$ & $\mathbf{1 2}$ & $\mathbf{0}$ & $\mathbf{2}$ & $*$ \\
number accepted, $\boldsymbol{p}=\mathbf{0 . 6}$ & $\mathbf{0}$ & $\mathbf{0}$ & $\mathbf{0}$ & $\mathbf{1 8}$ & $\mathbf{2}$ & $\mathbf{0}$ & $\mathbf{0}$ & $\mathbf{0}$ & $\mathbf{0}$ & $*$ \\
number rejected, $\boldsymbol{p}=\mathbf{0 . 6}$ & $\mathbf{0}$ & $\mathbf{0}$ & $\mathbf{2}$ & $\mathbf{2 0}$ & $\mathbf{8}$ & $\mathbf{2}$ & $\mathbf{0}$ & $\mathbf{0}$ & $\mathbf{0}$ & $*$ \\
number accepted, $\boldsymbol{p}=\mathbf{0 . 8}$ & $\mathbf{0}$ & $\mathbf{0}$ & $\mathbf{2 0}$ & $\mathbf{1 8}$ & $\mathbf{0}$ & $\mathbf{0}$ & $\mathbf{0}$ & $\mathbf{0}$ & $\mathbf{0}$ & $*$ \\
number rejected, $\boldsymbol{p}=\mathbf{0 . 8}$ & $\mathbf{0}$ & $\mathbf{0}$ & $\mathbf{1 8}$ & $\mathbf{1 6}$ & $\mathbf{1 0}$ & $\mathbf{4}$ & $\mathbf{0}$ & $\mathbf{0}$ & $\mathbf{0}$ & $*$ \\
\hline
\end{tabular}




\section{References}

1. Binmore, K.; McCarthy, J.; Ponti, G.; Samuelson, L.; Shaked, A. A backward induction experiment. J.Econ. Theory 2002, 104, 48-88.

2. Binmore, K.; Shaked, A.; Sutton, J. A further test of noncooperative bargaining theory: A reply. Am. Econ. Rev. 1988, 78, 837-839.

3. Cohen, M.; Jaffray, J.-Y.; Said, T. Experimental comparison of individual behavior under risk and under uncertainty for gains and for losses. Organ. Behav. Hum. Decis. Process. 1987, 39, 122.

4. Davis, D.; Holt, C. In Experimental Economics; Princeton University Press: Princeton, NJ, USA, 1993.

5. Fearon, J.D. Rationalist explanations for war. Int. Organ. 1995, 49, 379-414.

6. Fréchette, G.R.; Schotter, A. In The Methods of Modern Experimental Economics; Oxford University Press: Oxford, UK, forthcoming.

7. Goeree, J.K.; Palfrey, T.; Holt, C.A. Regular quantal response equilibrium. Exp. Econ. 2006, 8, 347-367.

8. Goeree, J.K.; Holt, C.A. Asymmetric inequality aversion and noisy behavior in alternating-offer bargaining games. Eur. Econ. Rev. 2000, 44, 1079-1089.

9. Güth, W.; Schmittberger, R.; Schwarze, B. An experimental analysis of ultimatum bargaining. J. Econ. Behav. Organ. 1982, 3, 367-388.

10. Holt, C.; Roth, A. The Nash equilibrium: A perspective. Proc. Natl. Acad. Sci. USA 2004, 101, 3999-4002.

11. Kahneman, D.; Tversky, A. Prospect theory: An analysis of decision under risk. In Econometrica 1979, 47, 263-292.

12. McKelvey, R. D.; Palfrey, T. R. Quantal response equilibrium for normal form games. In Games and Economic Behavior 1995, 10, 6-38.

13. Neelin, J.; Sonnenschein, H.; Spiegel, M. A further test of noncooperative bargaining theory: Comment. Am. Econ. Rev. 1988, 78, 824-836.

14. Powell, R. Bargaining in the shadow of power. Games Econ. Behav. 1996, 15, 255-289.

15. Powell, R. In Shadow of Power: States and Strategies in International Politics; Princeton University Press: Princeton, NJ, USA, 1999.

16. Samuelson, L. Economic theory and experimental economics. J. Econ. Lit. 2005, 43, 65-107.

17. Siegel, S.; Castellan, J. Nonparametric Statistics for the Behavioral Sciences; McGraw-Hill: New York, NY, USA, 1988.

18. Tomz, M.; Weeks, J. Public opinion and the democratic peace. Am. Polit. Sci. Rev. 2013, forthcoming.

19. Werner, S. Choosing demands strategically: The distribution of power, the distribution of benefits, and the risk of conflict. J. Confl. Resolut. 1999, 43,705-726.

(C) 2013 by the authors; licensee MDPI, Basel, Switzerland. This article is an open access article distributed under the terms and conditions of the Creative Commons Attribution license (http://creativecommons.org/licenses/by/3.0/). 